\title{
Effects of Wenyangbushen formula on the expression of VEGF, OPG, RANK and RANKL in rabbits with steroid-induced femoral head avascular necrosis
}

\author{
HONG-MEI SONG ${ }^{1,2}$, YING-CHEN WEI ${ }^{2}, \mathrm{NAN} \mathrm{LI}^{2}, \mathrm{BIN} \mathrm{WU}^{1}, \mathrm{NA} \mathrm{XIE}^{3}$, \\ KUN-MU ZHANG ${ }^{1}$, SHI-ZHONG WANG ${ }^{4}$ and HE-MING WANG ${ }^{2}$ \\ ${ }^{1}$ Department of Rehabilitation, Second Affiliated People's Hospital of Fujian Traditional Chinese Medicine University; \\ ${ }^{2}$ Orthopedic Department, Fujian Traditional Chinese Medicine University, Fuzhou, Fujian 350003; \\ ${ }^{3}$ Quality Control Department, Harrison International Peace Hospital, Hengshui, Hebei 053000; \\ ${ }^{4}$ Fujian Vocational College of Bioengineering, Fuzhou, Fujian 350002, P.R. China
}

Received November 15, 2014; Accepted August 4, 2015

DOI: $10.3892 / \mathrm{mmr} .2015 .4478$

\begin{abstract}
The present study aimed to investigate the effects of Wenyangbushen formula on the mRNA and protein expression levels of vascular endothelial growth factor (VEGF), osteoprotegerin $(\mathrm{OPG})$, receptor activator of nuclear factor $(\mathrm{NF})-\kappa \beta$ ligand (RANK), and RANK ligand (RANKL) in a rabbit model of steroid-induced avascular necrosis of the femoral head (SANFH). The present study also aimed to examine the potential mechanism underlying the effect of this formula on the treatment of SANFH. A total of 136 New Zealand rabbits were randomly divided into five groups: Normal group, model group, and three groups treated with the traditional Chinese medicine (TCM), Wenyangbushen decoction, at a low, moderate and high dose, respectively. The normal group and positive control group were intragastrically administered with saline. The TCM groups were treated with Wenyangbushen decoction at the indicated dosage. Following treatment for 8 weeks, the mRNA and protein expression levels of VEGF, OPG, RANK and RANKL in the femoral head tissues were determined using reverse transcription-quantitative polymerase chain reaction and western blot analyses, respectively. The data revealed that Wenyangbushen decoction effectively promoted the growth of bone cells, osteoblasts and chondrocytes, and prevented cell
\end{abstract}

Correspondence to: Mr. He-Ming Wang, Orthopedic Department, Fujian Traditional Chinese Medicine University, 1 Huatuo Road, Shangjie, Minhou, Fuzhou, Fujian 350003, P.R. China

E-mail:whm27@163.com

Mr. Shi-Zhong Wang, Fujian Vocational College of Bioengineering, 42 Zhongdian Road, Hongshan Bridge, Fuzhou, Fujian 350002, P.R. China

E-mail: 1365866912@qq.com

Key words: steroid-induced avascular necrosis of the femoral head, Wenyangbushen formula, vascular endothelial growth factor, OPG-RANK-RANKL signaling pathways apoptosis in the SANFH. The mRNA and protein expression levels of OPG and VEGF were increased, while the levels of RANK and RANKL were reduced in the necrotic tissue of the model group, compared with those in the normal rabbits. Wenyangbushen treatment prevented these changes, manifested by an upregulation in the expression levels of VEGF and OPG, and downregulation in the expression levels of RANK and RANKL in a dose-dependent manner. It was concluded that treatment with Wenyangbushen formula alleviated necrosis of the femoral head induced by steroids. It was observed to promote bone cell, osteoblast and chondrocyte growth, as well as prevent cell apoptosis. In addition, it upregulated the expression levels of OPG and VEGF, and inhibited the expression levels of RANK and RANKL. These results suggest the potential use of Wenyangbushen formula as a possible approach for the effective treatment of SANFH.

\section{Introduction}

Steroid-induced avascular necrosis of the femoral head, (SANFH) is a pathological process of femoral bone degeneration, induced by steroids, which can cause the death of the dynamic components of bone (1). Statistical investigations have demonstrated that the hormone is critical in non-traumatic osteonecrosis (2,3). At present, the etiology and pathogenesis of steroid-induced osteonecrosis remains to be fully elucidated. Emerging data has indicated that various mechanisms are involved, including stasis bone intraosseous hypertension due to venous stasis, osteoporosis, fat embolism, fatty degeneration and necrosis of bone cells, microvascular coagulation with intravenous vasoactive factors, arterial vascular damage due to the accumulation of immune complexes, apoptosis, bone marrow stromal stem cell adipogenic differentiation and gene polymorphism $(4,5)$. Vascular endothelial growth factor (VEGF) is a glycoprotein, which is characterized by its ability to promote angiogenesis and osteogenic differentiation (6-8). Osteoprotegerin (OPG) is a novel member of the tumor necrosis factor (TNF) receptor family. It can inhibit osteoclast differentiation and increase bone density function (9). 
Alternatively, receptor activator of nuclear factor $(\mathrm{NF})-\kappa \mathrm{B}$ (RANK) ligand (RANKL) can induce pre-osteoclast differentiation into functional osteoclasts and dose-dependently activate osteoclast maturation (10). It has been reported that RANKL is the only cytokine, which can induce osteoclast differentiation and development (11). RANK, in combination with RANKL, can induce the osteoclast precursors to differentiate, survive, integrate and inhibit the apoptosis of osteoclast cells (12). The RANKL/RANK/OPG pathway is critical in the regulation of osteoclast differentiation and bone resorption. Wenyangbushen formula is a prescription from the Orthopedics Department of the Second People's Hospital Affiliated to Fujian Traditional Chinese Medicine University (Fujian, China), and there is compelling evidence that Wenyangbushen decoction is efficacious in treating SANFH. Therefore, the present study was designed to investigate whether treatment with Wenyangbushen formula ameliorates SANFH via VEGF and the RANKL/RANK/OPG pathways.

\section{Materials and methods}

Preparation of Wenyangbushen formula. In the present study Wenyangbushen formula was produced using the following components: Morinda (15 g), epimedium (9 g), drynaria (9 g), deerhorn gelatin (6 g), Salvia miltiorrhiza (9 g), Radix curcumae (9 g), Panax pseudoginseng (3 g), Astragalus (15 g), Achyranthes $(9 \mathrm{~g})$ and licorice $(3 \mathrm{~g})$. All the materials were obtained from National Medicines Corporation, Ltd. (Beijing, China). The preparation was processed into a decoction containing the crude drug $(2.5 \mathrm{~g} / \mathrm{ml})$ by the Second People's Hospital of Fujian Province (Fujian, China).

Experimental animals and design. All the procedures and protocols complied with the Animal Management Rule of the Ministry of Public Health, China (doc. no. 55, 2001), and the experimental protocol was approved by the Animal Care and Use Committee of Fujian University of Traditional Chinese Medicine (Fuzhou, China). A total of 136 New Zealand rabbits were obtained from the Department of Laboratory Animal Science, Beijing University (Beijing, China). All animals were maintained under standardized lighting conditions $(12 \mathrm{~h}$ light-dark cycle) and temperature $\left(22 \pm 2^{\circ} \mathrm{C}\right)$. Normal diet and mineral water were administered ad libitum. The rabbits were randomly divided into the following five groups $(n=24$ rabbits in each group): Normal group, SANFH model group, and three traditional Chinese medicine (TCM) Wenyangbushen decoction groups, at low $(6.44 \mathrm{~g} / \mathrm{kg} \cdot \mathrm{d})$, moderate $(9.66 \mathrm{~g} / \mathrm{kg} \cdot \mathrm{d})$ and high $(12.88 \mathrm{~g} / \mathrm{kg} \cdot \mathrm{d})$ doses of the decoction. Following treatment with Wenyangbushen decoction for 8 weeks, the rabbits were anesthetized via intramuscular injection of xylazine and ketamine at doses of 2.2 and $21 \mathrm{mg} / \mathrm{kg}$ body weight, respectively. The femoral head tissues were then collected. At the end of experiment, the rabbits were sacrificed by sodium pentobarbital overdose $(100 \mathrm{mg} / \mathrm{kg}$; Dolethal, Vétoquinol Especialidades Veterinarias, S.A., Madrid, Spain).

Model establishment. The SANFH model was replicated using horse serum combined with methylprednisolone (Sigma-Aldrich, St. Louis, MO, USA), according to a study by Hofstaetter et al (13). Briefly, the rabbits were administered with $10 \mathrm{ml} / \mathrm{kg}$ horse serum via the ear vein and, after 3 weeks, $6 \mathrm{ml} / \mathrm{kg}$ horse serum was administered. After 2 weeks, methylprednisolone was intraperitoneally injected at a dose of $45 \mathrm{mg} / \mathrm{kg}$, once a day for three consecutive days. In addition, penicillin at 100,000 units/rabbit was administered, once a day for 7 days, to prevent infection. The control animals were injected with an equal volume of saline. After 4 weeks, the animal model was established.

Histological analysis of the femoral head tissues. Following the establishment of the animal model, two rabbits in the normal control group and four rabbits in the model group were randomly selected. Their femoral heads were removed, fixed with $4 \%$ paraformaldehyde (Sigma-Aldrich) for $48 \mathrm{~h}$ ( $\mathrm{pH} 7.4$ ) and decalcified with $10 \%$ EDTA-Tris buffer (Sigma-Aldrich). Paraffin sections at $4 \mu \mathrm{m}$ were stained with hematoxylin and eosin (HE; Sigma-Aldrich). The pathological changes of the femoral head were observed under an optical microscope (Olympus Corporation, Tokyo, Japan). The successfully established animal model was characterized by a reduction in bony trabeculae, disappearance of bone cells in the lacunae and an increase in empty bone lacuna.

Reverse transcription-quantitative polymerase chain reaction $(R T-q P C R)$ for detection of the mRNA expression levels of VEGF, OPG, RANK and RANKL. The mRNA levels of VEGF, OPG, RANK and RANKL in the left femoral head tissues were determined using a RT-qPCR system (Beckman Coulter, Fullerton, CA, USA). Total RNA was isolated using TRIzol reagent (Invitrogen; Thermo Fisher Scientific, Inc.), according to the manufacturer's protocol. Total RNA $(2 \mu \mathrm{g})$ was reverse-transcribed using a Superscript First Strand Synthesis System (Invitrogen; Thermo Fisher Scientific, Inc.) to generate complementary DNA (cDNA). cDNA was immediately reverse-transcribed from the isolated RNA. The sequences of the primers used are indicated in Table I. Primers were obtained from Invitrogen (Thermo Fisher Scientific, Inc.). The cDNA samples were amplified in a DNA thermal cycler under the following conditions: The mixture was annealed at $57^{\circ} \mathrm{C}$ for $30 \mathrm{sec}$, extended at $72^{\circ} \mathrm{C}$ for $45 \mathrm{sec}$, and denatured at $94^{\circ} \mathrm{C}$ for $30 \mathrm{sec}$, with a total of 30 cycles. This was followed by a final extension step at $72^{\circ} \mathrm{C}$ for $10 \mathrm{~min}$ to ensure complete product extension. PCR was performed in a volume of $20 \mu \mathrm{l}$ containing $2 \mu \mathrm{l}$ buffer (10X), $0.5 \mu \mathrm{l}$ deoxynucleotide triphosphates (10 mM), $0.5 \mu \mathrm{l}$ Taq enzyme, $0.5 \mu \mathrm{l}$ Primer F (10 mM), $0.5 \mu \mathrm{l}$ Primer R (10 mM), $14 \mu \mathrm{l} \mathrm{ddH}_{2} \mathrm{O}$ and $2 \mu \mathrm{l}$ DNA template. The qPCR products were resolved on a $1.5 \%$ agarose gel (Sigma-Aldrich) and analyzed on a white/ultraviolet transilluminator digital science and analysis system (14). The expression levels of the target genes were determined in triplicate.

Western blotting to determine the protein expression levels of VEGF, OPG, RANK and RANKL. The right femoral head tissues were collected and lysed in radioimmunoprecipitation assay buffer (Sigma-Aldrich), containing $50 \mathrm{mM}$ Tris- $\mathrm{Cl}$ (pH 8.0), $150 \mathrm{mM} \mathrm{NaCl}, 1 \%$ Nonidet P-40, 0.5\% sodium deoxycholate, $0.1 \% \mathrm{SDS}, 100 \mu \mathrm{g} / \mathrm{ml}$ phenylmethyl-sulfonyl fluoride and $2 \mu \mathrm{g} / \mathrm{ml}$ aprotinin. The suspension was incubated on ice and then centrifuged $\left(14,000 \mathrm{~g}, 10 \mathrm{~min}, 4^{\circ} \mathrm{C}\right)$. The soluble fraction was stored at $-80^{\circ} \mathrm{C}$. Protein concentrations were 
Table I. Primers used for reverse transcription-quantitative polymerase chain reaction analysis.

\begin{tabular}{lll}
\hline Gene & \multicolumn{1}{c}{ Forward sequence (5'-3') } & \multicolumn{1}{c}{ Reverse sequence (5'-3') } \\
\hline VEGF & CGTTTCCTTCCTCATCTCCTC & GCTTGTTCCTCCTTCTTGCTC \\
OPG & ACAATGAACAAGTGGCTGTGCTG & CGGTTTCTGGGTCATAATGCAAG \\
RANK & TTCAGGTTTGCTGTTCCTACA A & CGCCGTTTTATCCTCTCTACAC \\
RANKL & GCAGCATCGCTCTGTTCCTGTA & GCATGAGTCAGGTAGTGCTTCTGTG \\
$\beta$-actin & AGACCACCTTCAACTCGATCAT & ACTCGTCATACTCCTGCTTGCT
\end{tabular}

VEGF, vascular endothelial growth factor; OPG, vsteoprotegerin; RANK, receptor activator of nuclear factor $\kappa \beta$; RANKL, RANK ligand.
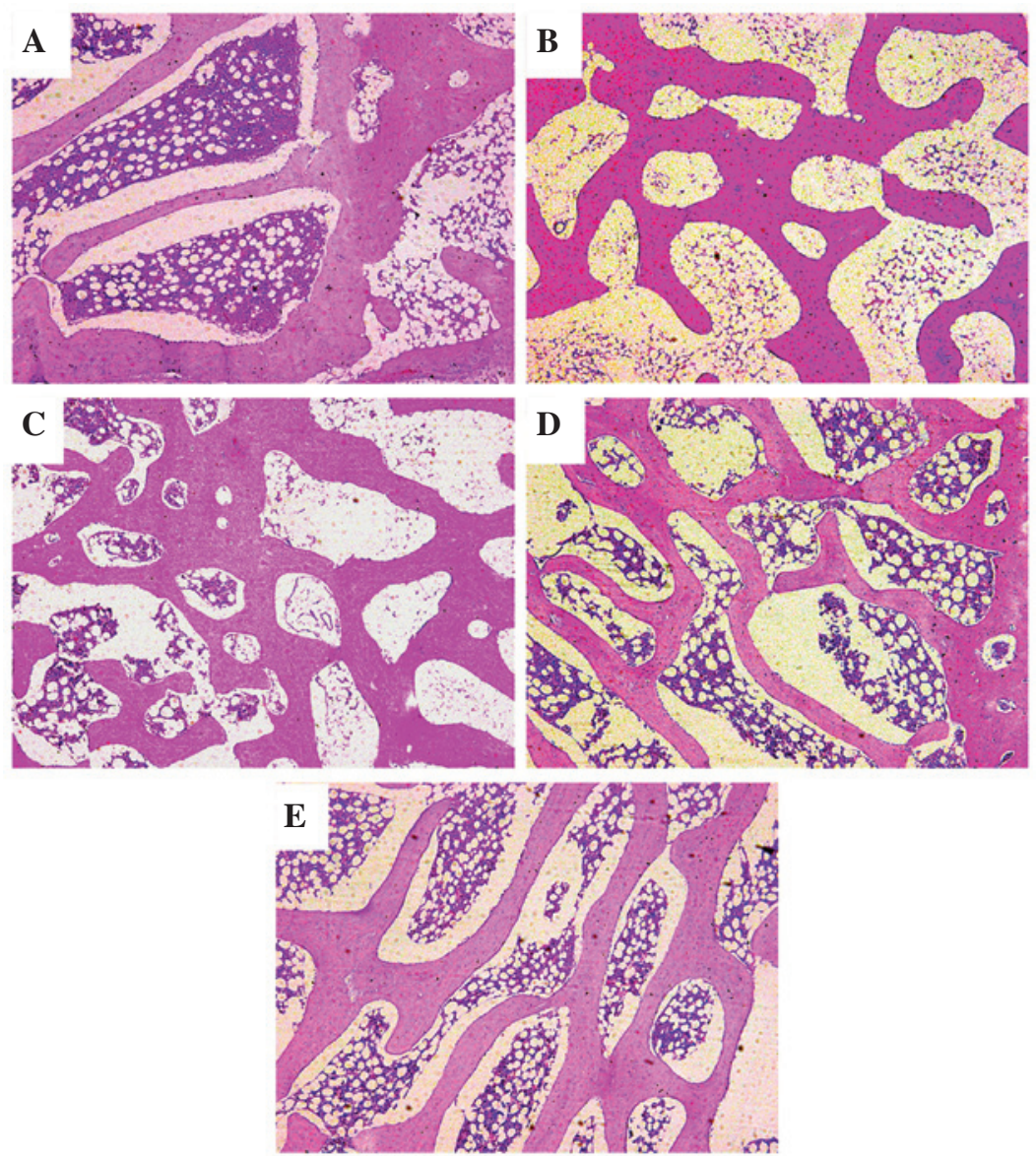

Figure 1. Effects of Wenyangbushen decoction on bone histology in the steroid-induced avascular necrosis of the femoral head model. (A) Normal group; (B) model group; (C) low dose-treated group; (D) moderate dose-treated group; (E) high dose-treated group. (Hematoxylin and eosin staining; magnification, $\mathrm{x} 50$ ).

measured using a Bradford assay with bovine serum albumin as a standard. Proteins $(40 \mu \mathrm{g})$ were run on a $12 \%$ SDS-PAGE gel (for VEGF and RANKL) or 8\% SDS-PAGE gel (for RANK and $\mathrm{OPG}$ ), and then transferred onto polyvinylidene fluoride membranes. Following incubation with $10 \%$ non-fat milk for $1 \mathrm{~h}$, the membranes were probed with primary antibodies overnight at $4^{\circ} \mathrm{C}$ and then incubated with goresradish peroxidase-labeled anti-rabbit secondary antibodies (1:2,000; Santa Cruz Biotechnology, Inc., Dallas, TX, USA). The protein levels were normalized using $\beta$-actin as a loading control. The relative optical density of the protein bands was measured using a Zeineh Laser Densitometer (Biomed Instruments, Inc., Fullerton, CA, USA) following subtracting the film background. Incubation with primary antibodies was then performed at following dilutions: Primary antibodies were used as follows: Mouse monoclonal anti-VEGF (1:500, cat. no. ab1316; Abcam, Cambridge, UK); rabbit polyclonal anti-OPG (1:400; cat. no. BA1475-1; Boster Biological Engineering Co. Ltd., Wuhan, China), rabbit polyclonal anti-RANK (1:300; cat. no. BA1323; Boster Biological Engineering Co., Ltd.) and rabbit polyclonal anti-RANKL (1:650; cat. no. ab22113; Abcam). The intensity of the bands was quantified using densitometry. The resulting blots are representative of at least three experiments.

Statistical analysis. All values are expressed as the mean \pm standard error of the mean, unless otherwise indi- 

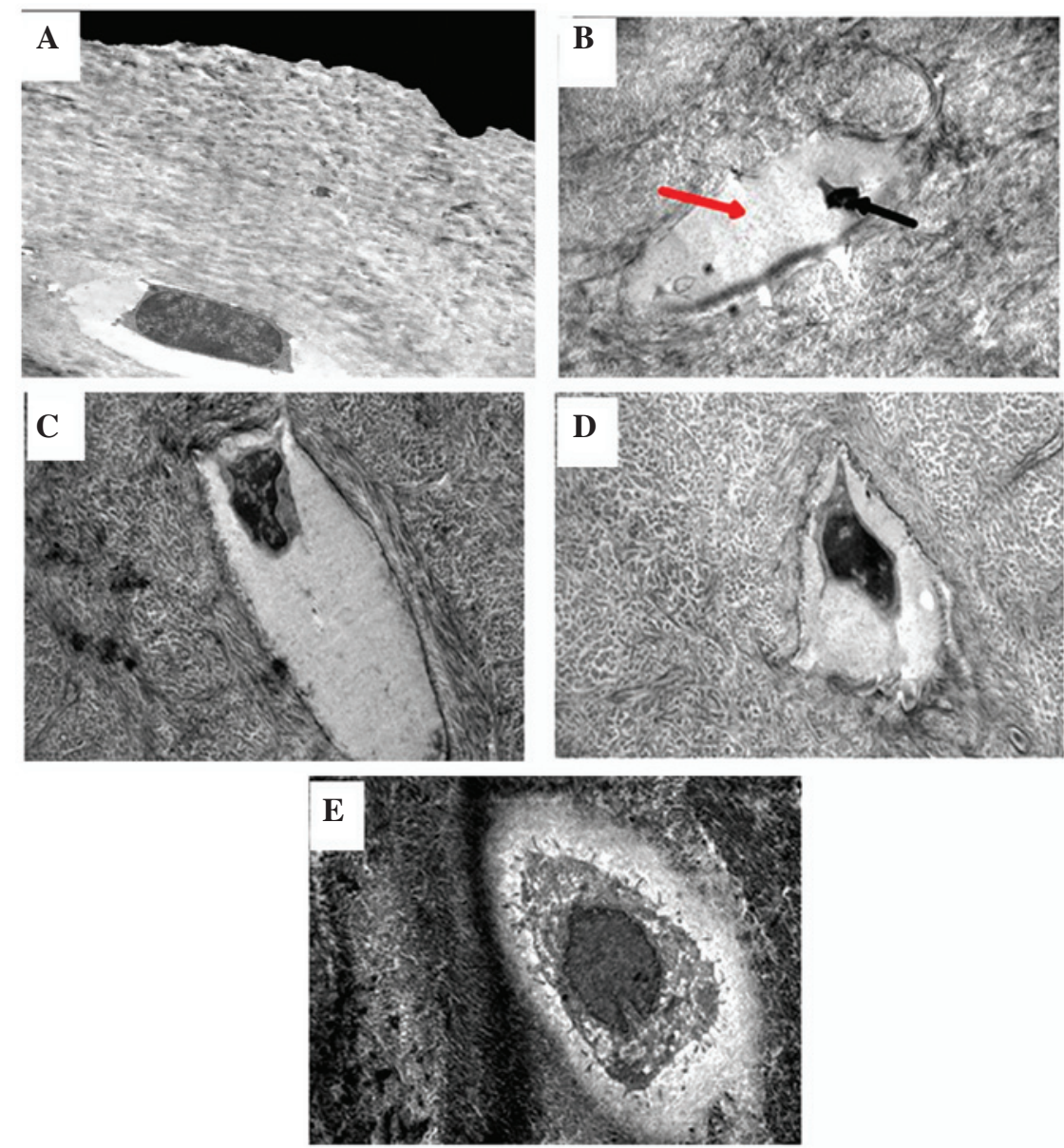

Figure 2. Effects of Wenyangbushen decoction on bone cell ultrastructure in the steroid-induced avascular necrosis of the femoral head model. The model animals exhibited apoptotic bone cells (red arrow) and empty bone lacuna formation (black arrow). (A) Normal group; (B) model group; (C) low dose-treated group; (D) moderate dose-treated group; (E) high dose-treated group. (Electron microscope, magnification, x10,000).

cated. Group comparisons were performed using Student's t-test (two sample test) or one-way analysis of variance. A Mann-Whitney U test was used when the variance was not normally distributed. $\mathrm{P}<0.05$ was considered to indicate a statistically significant difference. Statistical analysis was performed using SPSS 17.0 software (SPSS, Inc., Chicago, IL, USA).

\section{Results}

Histological analysis using HE staining. In the normal control group, the cartilage thickness of the femoral head layer was normal. The cartilage surface was smooth and lined with dense and regular bony trabeculae. The bone structure was integral and the cells in each layer were evenly distributed. Cartilage cells were arranged regularly. Bone cells with normal morphology were clearly visible, located in the central position of the bone lacuna, with occasional, scattered, empty lacunae. The bone marrow cavity was rich in bone marrow cells, and a small number of fat cells with normal morphology were noted (Fig. 1A). In the model group, the cartilage of the femoral head layer was thinner, compared with that of the normal group (Fig. 1B). Areas of the cartilage surface appeared exfoliated with cracks. The bony trabeculae were loose, irregular, slender and sparse, and even broken. All types of cells were scattered and sparsely distributed. The numbers of chondrocytes were reduced substantially and often clustered together. The number of bone cells was decreased, compared with the normal model, and empty lacunae were observed. The numbers of adipose cells were increased and were hypertrophied, degenerate and becoming necrotic. By contrast, treatment with Wenyangbushen decoction at the indicated concentrations (Fig. 1C-E) led to improvements in these pathological changes.

In addition, the number of empty lacunae in the normal group and in the model group were counted under a light microscope. The ratios of empty lacunae in the normal group and model group were $9.5 \pm 1.152$ and $21 \pm 1.078 \%$, respectively. The difference between the two groups was statistically significant $(\mathrm{P}<0.01)$. Wenyangbushen decoction treatment decreased the number of empty lacunae.

Characteristics of bone cell ultrastructure visualized under an electron microscope. In the normal control group (Fig. 2A), the bone cells were predominantly elongated-oval shaped, located in the bone lacunae and were plump in appearance. There were several prominentia on the cell surface. The nuclear heterochromatin was uniform. The mitochondria and rough endoplasmic reticulum were well-developed. The number of glycogen granules were rich in the cytoplasm. A 
A

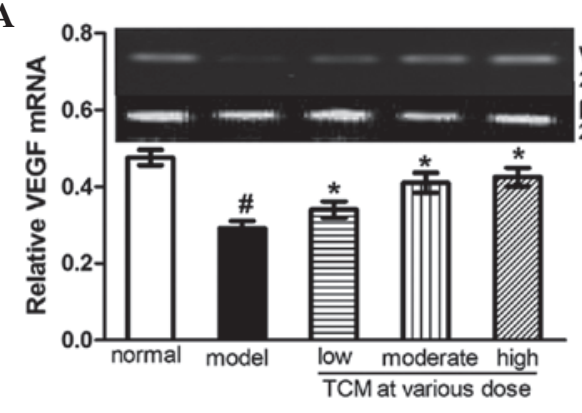

C

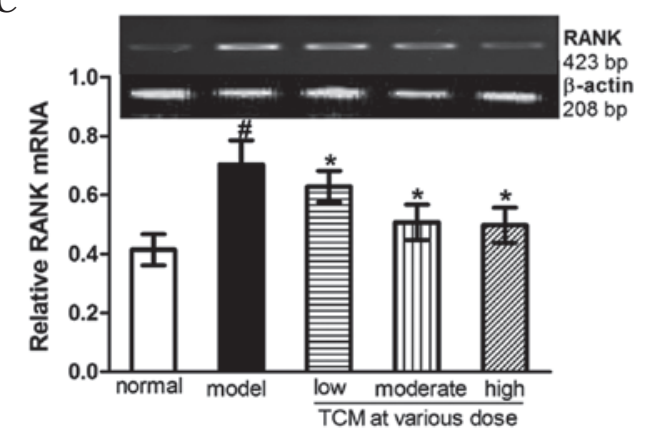

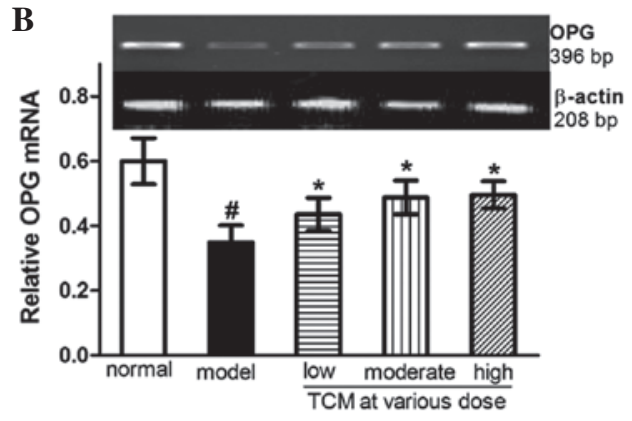

D

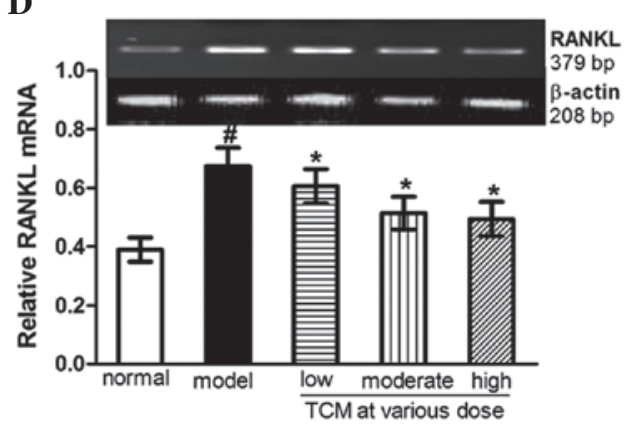

Figure 3. Effects of Wenyangbushen decoction on the mRNA expression levels of VEGF, OPG, RANK and RANKL in the steroid-induced avascular necrosis of the femoral head animal model. The expression of (A) VEGF, (B) OPG, (C) RANK and (D) RANKL were determined using reverse transcription-quantitative polymerase chain reaction. $\beta$-actin served as an endogenous control. Results are presented as the mean \pm standard error of the mean of three independent experiments in triplicate. ${ }^{\prime \prime} \mathrm{P}<0.05$, vs. normal control; ${ }^{*} \mathrm{P}<0.05$, vs. model. Low dose, $6.44 \mathrm{~g} / \mathrm{kg} \cdot \mathrm{d}$; moderate dose, $9.66 \mathrm{~g} / \mathrm{kg} \cdot \mathrm{d} ; \mathrm{high}$ dose, $12.88 \mathrm{~g} / \mathrm{kg} \cdot \mathrm{d}$; TCM, traditional Chinese medicine (Wenyangbushen decoction); VEGF, vascular endothelial growth factor; OPG, osteoprotegerin; RANK, receptor activator of nuclear factor- $\kappa \beta$; RANKL, RANK ligand.

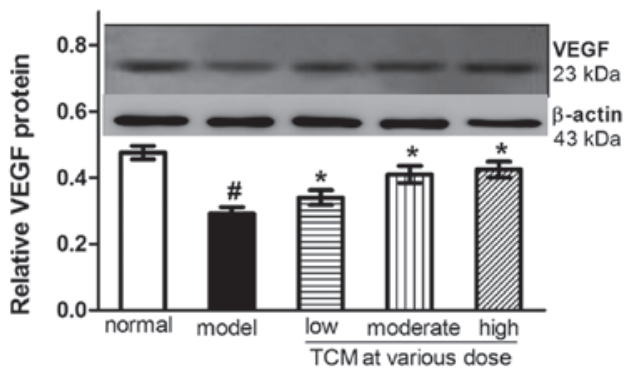

C

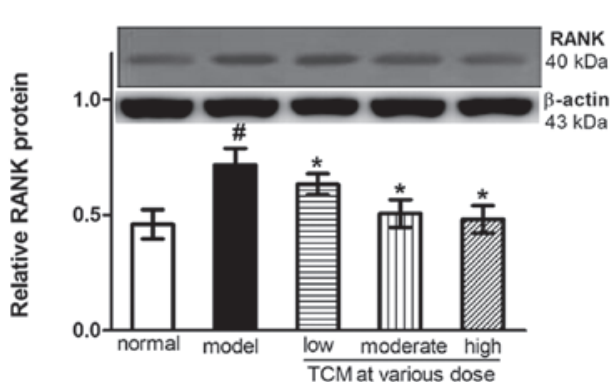

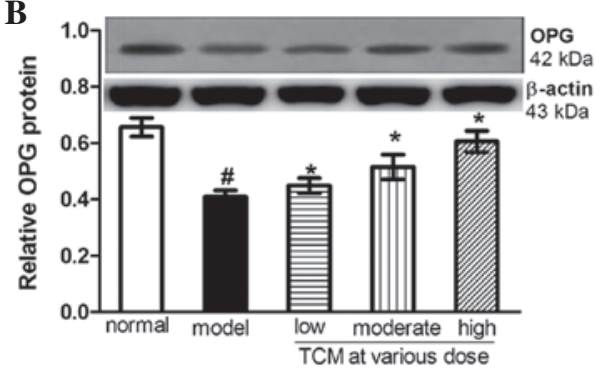

D

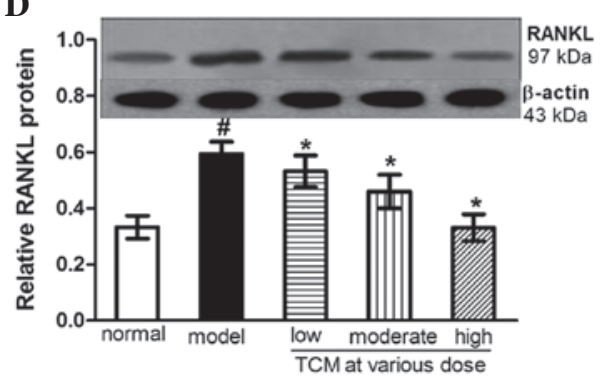

Figure 4. Effects of Wenyangbushen decoction on the protein expression levels of VEGF, OPG, RANK and RANKL in the steroid-induced avascular necrosis of the femoral head animal model. The expression levels of (A) VEGF, (B) OPG, (C) RANK and (D) RANKL were determined using western blotting. $\beta$-actin served as a loading control. Results are presented as the mean \pm standard error of the mean of three independent experiments in triplicate. ${ }^{~} \mathrm{P}<0.05$, vs. normal control; " $\mathrm{P}<0.05$, vs. model. Low dose, $6.44 \mathrm{~g} / \mathrm{kg} \cdot \mathrm{d}$; moderate dose, $9.66 \mathrm{~g} / \mathrm{kg} \cdot \mathrm{d}$; high dose, $12.88 \mathrm{~g} / \mathrm{kg} \cdot \mathrm{d}$; TCM, traditional Chinese medicine (Wenyangbushen decoction); VEGF, vascular endothelial growth factor; OPG, 0steoprotegerin; RANK, receptor activator of nuclear factor- $\kappa \beta$; RANKL, RANK ligand.

small number of lysosomes were observed. The peripheral collagen fibers were densely distributed and their composition was regular and uniform.
In the model group (Fig. 2B), the bone cells exhibited karyopyknosis, chromatin aggregation and nuclear fragmentation. The bone cells were squeezed aside by large lipid 
droplets, and exhibited various degrees of necrosis, apoptosis and empty bone lacuna formation. The collagen fibers were thick and disorganized.

In the low dose TCM-treatment group (Fig. 2C), the bone cells were increasedin number, compared with the model group. The arrangement of rough endoplasmic reticulum, mitochondria and collagen fibers were also improved, compared with the model group. In the moderate- and high-dose TCM treatment groups (Fig.2D and E), the bone cells were further increased in number and the organelles were well-organized and abundant in mitochondria and rough endoplasmic reticulum. The distribution of collagen fibers were dense and well arranged.

Wenyangbushen decoction upregulates the mRNA expression levels of VEGF and OPG, and downregulates the $m R N A$ expression levels of RANK and RANKL in the SANFH model. Compared with the normal control rabbits, the mRNA levels of VEGF and OPG were decreased (Fig. 3A and B), while those of RANK and RANKL were increased (Fig. 3C and D), in the SANFH model group. Wenyangbushen treatment prevented these changes, which exhibited upregulation in the mRNA expression levels of VEGF and OPG, and downregulation in the mRNA expression levels of RANK and RANKL, compared with the model group. These changes occurred in a concentration-dependent manner. In the presence of a high dose of the Wenyangbushen decoction, the mRNA expression levels of VEGF and OPG were increased by 45.5 and $42.1 \%$, respectively, and the expression levels of RANK and RANKL were attenuated by 29.4 and $26.7 \%$ respectively, compared with those in the model group (Fig. 3).

Effects of Wenyangbushen decoction on the protein expression levels of VEGF, OPG, RANK and RANKL in the SANFH model. Consistent with the results observed in the examination of the mRNA expression levels, the protein expression levels of VEGF and OPG (Fig. 4 A and B) were also reduced, while those of RANK and RANKL (Fig. 4 C and D) were increased in the SANFH group, compared with the model group. The protein expression levels of VEGF and OPG were markedly enhanced, while those of RANK and RANKL were inhibited by Wenyangbushen treatment, compared with the model group, and this also occurred in a dose-dependant manner. Compared with the SANFH model counterparts, treatment with a high-dose of Wenyangbushen increased the expression of VEGF 1.43-fold and OPG 1.48-fold. Compared with the SANFH model group, the protein expression levels of RANK and RANKL were decreased by 32.9 and $44.3 \%$, respectively.

\section{Discussion}

The pathogenesis of steroid induced femoral head necrosis is complex and remains to be fully elucidated. Emerging data suggests that SANFH is a multifactorial disease, which is predominantly associated with bone structure destruction, cell necrosis and vascular stasis. Therefore, the fundamental method of treatment for SANFH is to promote the formation of bone and regeneration of vessels $(15,16)$, and the effective management of these two aspects is a priority. The fundamental principle of Warming Yang and Tonifying Kidney was established, according to the traditional Chinese medicine theory 'the kidney governs the bones and engenders marrow' (17). Our previous study indicated that Wenyangbushen decoction promotes the differentiation of bone marrow stromal cells, chondrocyte proliferation and ultimately prevents the development of SANFH (18).

In the process of bone formation and absorption, RANKL, RANK and OPG act in a combination to regulate the differentiation of osteoblasts and osteoclasts. When various stimulating factors act on osteoblasts or stromal cells to express RANKL on their surface, they identify specifically the osteoclast precursors and bind RANK receptor on the cell membrane (19). In the presence of M-CSF, the RANK-RANKL signal can be transduced into the cells and stimulate the maturation of osteoclast precursors into osteoclasts $(20,21)$. In addition, the actin ring in mature osteoclasts develops and is activated via the RANK-RANKL pathway, which can promote bone resorption, whereas OPG can competitively inhibit the binding of RANKL and RANK, and inhibits the biological effects of RANK-RANKL.

OPG also binds RANKL/RANK to form a trimer, which can directly inhibit the function of RANKL/RANK. RANKLor RANK-deficient mice exhibit abnormal bone resorption and severe bone sclerosis. OPG-deficient mice also develop osteoporosis following birth, which can be alleviated by treatment with recombinant OPG (22). Therefore, the RANKL/RANK/OPG pathway is a key loop regulator of osteoblast and osteoclast differentiation, as well as bone resorption (23). The data of the present study suggested that the expression levels of RANK and RANKL were significantly increased, while that of OPG was decreased in the femoral head tissues of the model group, compared with the normal control. However, treatment with Wenyangbushen decoction was observed to reverse these effects by inhibiting the expression of RANK and RANKL, while upregulating the expression of OPG. This led to the inhibition of osteoclast differentiation and function, as well as the promotion of osteoblast activity.

The expression of VEGF in bone tissue is affected by hormones. Decreased expression of VEGF can lead to a reduction in angiogenesis in bone tissues and a lack of blood supply to the femoral head, leading eventually to femoral head necrosis (24). Lee et al reported that the VEGF gene promotes angiogenesis and stimulates bone growth in SANFH (25). Similarly, the results of the present study suggested that the expression of VEGF in the model group was significantly decreased, compared with the normal control group. Additionally, its expressionlevel was substantially increased by Wenyangbushen decoction treatment, which indicated that VEGF is critical in SANFH.

In conclusion, the results of the present study demonstrated that Wenyangbushen decoction effectively promoted bone cell, osteoblast and chondrocyte growth, and prevented cell apoptosis. The mechanism underlying these effects may be associated with it inhibiting the expression of RANK and RANKL, and promoting the expression of OPG and VEGF in SANFH. Accordingly, Wenyangbushen decoction may be considered a potential candidate drug for SANFH treatment.

\section{Acknowledgements}

This study was supported by grants from the National Natural Science Foundation of China (grant. no. 81173283) and Project of Fujian Province Educational Department (grant. no. JA13156). 


\section{References}

1. Beckmann R, Shaheen H, Kweider N, Ghassemi A, Fragoulis A, Hermanns-Sachweh B, Pufe T, Kadyrov M and Drescher W: Enoxaparin prevents steroid-related avascular necrosis of the femoral head. Scientific World Journal 2014: 347813, 2014.

2. Sheng H, Sheng CJ, Cheng XY, Zhang G, Lee KM, Leung KS, Qu S and Qin L: Pathomorphological changes of bone marrow adipocytes in process of steroid-associated osteonecrosis. Int J Clin Exp Pathol 6: 1046-1050, 2013.

3. Bekler H, Uygur AM, Gökçe A and Beyzadeoğlu T: The effect of steroid use on the pathogenesis of avascular necrosis of the femoral head: An animal model. Acta Orthop Traumatol Turc 41: 58-63, 2007 (In Turkish).

4. Steffen RT, Athanasou NA, Gill HS and Murray DW: Avascular necrosis associated with fracture of the femoral neck after hip resurfacing: Histological assessment of femoral bone from retrieval specimens. J Bone Joint Surg Br 92: 787-793 2010.

5. Tong P, Wu C, Jin H, Mao Q, Yu N, Holz JD, Shan L, Liu H and Xiao L: Gene expression profile of steroid-induced necrosis of femoral head of rats. Calcif Tissue Int 89: 271-284, 2011.

6. Varoga D, Drescher W, Pufe M, Groth G and Pufe T: Differential expression of vascular endothelial growth factor in glucocorticoid-related osteonecrosis of the femoral head. Clin Orthop Relat Res 467: 3273-3782, 2009.

7. Yeh LC and Lee JC: Osteogenic protein-1 increases gene expression of vascular endothelial growth factor in primary cultures of fetal rat calvaria cells. Mol Cell Endocrino 153: 113-124, 1999.

8. Wang G, Zhang CQ, Sun Y, Feng Y, Chen SB, Cheng XG and Zeng BF: Changes in femoral head blood supply and vascular endothelial growth factor in rabbits with steroid-induced osteonecrosis. J Int Med Res 38: 1060-1069, 2010.

9. Simonet WS, Lacey DL Cunstan CR, Kelley M, Chang MS, Lüthy R, Nguyen HQ, Wooden S, Bennett L, Boone T, et al: Osetoprotegerin: A novel secreted protein involved in the regulation of bone density. Cell 89: 309-319, 1997.

10. Shiotani A, Takami M, Itoh K, Shibasaki Y and Sasaki T: Regulation of osteoclast differentiation and function by recep to ractivator of NFkB ligand and osteoprotegerin. Anat Rec 268: 137-146, 2002

11. Boyce BF and Xing L: Functions of RANKL/RANK/OPG in bone modeling and remodeling. Arch Biochem Biophys 473: 139-146, 2008 .
12. Boyce BF and Xing L: The RANKL/RANK/OPG pathway. Curr Osteoporos Rep 5: 98-104, 2007.

13. Hofstaetter JG, Wang J, Yan J and Glimcher MJ: The effects of alendronate in the treatment of experimental osteonecrosis of the hip in adult rabbits. Osteoarthritis Cartilage 17: 362-370, 2009.

14. Zhong X, Lin J, Zhou J, Xu W and Hong Z: Anti-proliferative effects of qianliening capsules on prostatic hyperplasia in vitro and in vivo. Mol Med Rep 12: 1699-1708, 2015.

15. Seamon J, Keller T, Saleh J and Cui Q: The pathogenesis of nontraumatic osteonecrosis. Arthritis 2012: 601763, 2012.

16. Yang L, Boyd K, Kaste SC, Kamdem L, Rahija RJ and Relling MV: A mouse model for glucocorticoid-induced osteonecrosis: effect of a steroid holiday. J Orthop Res 7: 169-175, 2009.

17. Liu R, Kang X, Xu L, Nian H, Yang X, Shi H and Wang X: Effect of the combined extracts of Herba epimedii and Fructus ligustri lucidi on sex hormone functional levels in osteoporosis rats. Evid Based Complement Alternat Med, 2015: 184802, 2015.

18. Chen WH and Wang HM: Experimental research progress of warming yang and reinforcing kidney of Chinese medicine to promote the differentiation of bone marrow stromal cells. Zhongguo Gu Shang 24: 352-356, 2011 (In Chinese).

19. Bai YD, Yang FS, Xuan K, Bai YX and Wu BL: Inhibition of RANK/RANKL signal transduction pathway: a promising approach for osteoporosis treatment. Med Hypotheses 71: 256-258, 2008.

20. Kim HK, Morgan-Bagley S and Kostenuik P: RANKL inhibition: A novel strategy to decrease femoral head deformity after ischemic osteonecrosis. J Bone Miner Res 21: 1946-1954, 2006.

21. Wittrant Y, Theoleyre S, Couillaud S, Dunstan C, Heymann D and Rédini F: Relevance of an in vitro osteoclastogenesis system to study receptor activator of NF-kB ligand and osteoprotegerin biological activities. Exp Cell Res 293: 292-301, 2004.

22. Geusens P: The role of RANK ligand/osteoprotegerin in rheumatoid arthritis. Ther Adv Musculoskelet Dis 4: 225-233, 2012.

23. Mandelin J, Li TF, Liljeström M, Kroon ME, Hanemaaijer R, Santavirta $S$ and Konttinen YT: Imbalance of RANKL/RANK/OPG system in interface tissue in loosening of total hip replacement. J Bone Joint Surg Br 85: 1196-1201, 2003.

24. Varoga D, Drescher W, Pufe M, Groth G and Pufe T: Differential expression of vascular endothelial growth factor in glucocorticoid-related osteonecrosis of the femoral head. Clin Orthop Relat Res 467: 3273-3282, 2009.

25. Lee YJ, Lee JS, Kang EH, Lee YK, Kim SY, Song YW and Koo KH: Vascular endothelial growth factor polymorphisms in patients with steroid-induced femoral head osteonecrosis. J Orthop Res 30: 21-27, 2012. 